\title{
DARBOUX EQUATIONS IN EXTERIOR DOMAINS *
}

\author{
SAOUSSEN KALLEL-JALLOULI ${ }^{\dagger}$
}

Abstract. We give sufficient conditions ensuring existence and regularity of a radial solution to the following equation

$$
\begin{aligned}
& \operatorname{det}\left(\phi_{i j}\right)=F(|x|, \phi,|\nabla \phi|), \text { in } \Omega \\
& \phi_{\mid \partial \Omega}=c
\end{aligned}
$$

when $\Omega$ is an exterior domain.

1. Introduction. In this work, we consider the Dirichlet problem for real Monge-Ampère equations in exterior domains. More precisely, let $B \subset \mathbb{R}^{n}$ be an open ball, centered at the origin, that can be supposed, without loss of generality, to be the unit ball . Our purpose is to establish the existence of radial, convex solution $u \in C^{2}\left(\mathbb{R}^{n} \backslash B\right)$ of radially symmetric Monge-Ampère equation

$$
\left\{\begin{array}{l}
\operatorname{det}\left(\phi_{i j}\right)=F(|x|, \phi,|\nabla \phi|), \quad \text { in } \mathbb{R}^{n} \backslash \bar{B} \\
\phi_{\mid \partial B}=c
\end{array}\right.
$$

where $F$ is a nonnegative continuous function. As usual, $|x|$ denotes the Euclidean length of $x=\left(x_{1}, \ldots, x_{n}\right)$ and $n$ is (all over this paper) the dimension of our Euclidean space. Additional hypothesis on $F$ are described in $\S 2$.

When $\Omega$ is a strictly convex domain, this problem has received considerable study. Not many results are known about the solutions in unbounded domains. In the case when $F>0$, F.Finster and O.C. Schnürer [2] proved the existence of smooth, strictly convex solution to (1) under some restrictions on $F$. We can also cite the work of T. Kusano and Ch.A. Swanson [3] related to radially symmetric two-dimensional elliptic Monge-Ampère equations.

Our attention will be directed toward the construction of radial solutions $u(x)=$ $u(t)$ of (1), $t=|x|$. Direct computation (see [1]), shows that solving the equation (1) in $C^{2}$ is equivalent to solving the ordinary differential equation

$$
\left\{\begin{array}{l}
{\left[\left(y^{\prime}\right)^{n}\right]^{\prime}=n t^{n-1} F\left(t, y, y^{\prime}\right), \quad \text { if } t>1} \\
y(1)=c
\end{array}\right.
$$

Without loss of generality, we can take $c=0$.

If we take as initial condition $y^{\prime}(1)=0$, we can easily transform (2) into the following integro-differential equation

$$
y(r)=\int_{1}^{r}\left[\int_{1}^{\rho} n t^{n-1} F\left(t, y(t), y^{\prime}(t)\right) d t\right]^{\frac{1}{n}} d \rho
$$

\footnotetext{
*Received April 22, 2003; accepted for publication May 18, 2004.

$\dagger$ Faculté des Sciences, Campus Universitaire, 1060, Tunisie (Saoussen.Kallel@fst.rnu.tn).
} 
EXAMPLE. Let $F(r)=(r-1)^{n-1-\varepsilon} r^{1-n}$, with $\varepsilon>0$ small enough. Then, $u^{\prime}(r)=\left[\frac{n}{n-\varepsilon}\right]^{\frac{1}{n}}(r-1)^{1-\frac{\varepsilon}{n}}$. In this case $F \in C^{0}$, but $u \notin C^{2}$.

This example shows that even when $F$ depends only on $r$, it may not yield a $C^{2}$ solution, if $F$ is allowed to vanish in the domain. This implies that we should place some restrictions on $F$.

Throughout this work, $F$ satisfies some hypothesis be selected from the following list:

$\left(H_{1}\right)$ :

i) $F(t, y, z)$ is a nonincreasing function with respect to both $y$ and $z$ for each fixed $(t, z)$ and $(t, y)$, respectively.

ii) $\int_{1}^{+\infty} t^{n-1} F(t, 0,0) d t<+\infty$

$\left(H_{2}\right): F(t, y, z) \leq C_{0} t^{-n-\alpha}|y|^{\beta}|z|^{\theta}$, with $\beta \geq 0, \alpha>\beta, \theta \geq 0$ and $C_{0} \leq \frac{\alpha-\beta}{n}$ if $\beta+\theta=n$.

$\left(H_{3}\right)$ :

i) $F(t, y, z)$ is a nondecreasing function with respect to both $y$ and $z$ for each fixed $(t, z)$ and $(t, y)$, respectively.

ii) There exists a constant $a>0$ such that

$$
\int_{1}^{+\infty} n t^{n-1} F(t,(t-1) a, a) d t \leq a^{n}
$$

$\left(H_{4}\right): F(t, y, z)=(t-1)^{l} \widetilde{F}(t, y, z)$, with $\widetilde{F}(1, y, 0) \neq 0$, for $y \geq 0, l \geq n-1$, $\widetilde{F} \in C^{0}$.

An example of a Monge-Ampère equation satisfying $\left(H_{3}\right)$ is the Gauss curvature equation

$$
\left\{\begin{array}{l}
\operatorname{det}\left(u_{i j}\right)=p(|x|) u^{\gamma}\left(1+|\nabla u|^{2}\right)^{\delta}, \quad x \in \mathbb{R}^{n} \backslash B \\
u_{\mid \partial B}=1
\end{array}\right.
$$

with $\gamma, \delta \geq 0,2 \delta+\gamma<n$ and $p$ is a non-négative function satisfying:

$$
\int_{1}^{+\infty} t^{n+\gamma-1} p(t) d t<+\infty
$$

In the following, $\widetilde{F}$ is used as introduced in $\left(H_{4}\right)$. We shall prove

Theorem A. If $\left(H_{4}\right)$ and either $\left(H_{1}\right),\left(H_{2}\right)$ or $\left(H_{3}\right)$ holds, equation (1) has an infinitude of radial convex solutions $u \in C^{2}$ such that $\frac{u(x)}{|x|}$ has a positive finite limit at $\infty$.

Theorem B. If we suppose, in addition to the hypothesis of Theorem A, that

$$
\widetilde{F} \in C^{k}\left(\left(\mathbb{R}^{n} \backslash B\right) \times \mathbb{R}^{2}\right)
$$

and 


$$
\text { either } \frac{l+1}{n} \in \mathbb{N} \text { or } \frac{l+1}{n} \geq k+1
$$

then the solutions given by theorem $\mathrm{A}$ are in $C^{k+2}$

2. Proof of theorem A. To prove the existence of a radially symmetric convex solution to the problem (1), we need to introduce the Frechet space $C^{1}$ of all continuously differentiable functions in $[1,+\infty$ [, with the topology of uniform convergence of functions and their first derivatives on compact intervals. Consider now the closed convex subset $\mathcal{K}_{R}$ of $C^{1}$

$$
\mathcal{K}_{R}=\left\{y \in C^{1} \mid y(1)=0,0 \leq y^{\prime}(t) \leq R\right\}
$$

and the operator $T: \mathcal{K}_{\mathcal{R}} \rightarrow C^{1}$ defined by

$$
T(y)(r)=\int_{1}^{r}\left[\int_{1}^{\rho} n t^{n-1} F\left(t, y(t), y^{\prime}(t)\right) d t\right]^{\frac{1}{n}} d \rho, r \geq 1
$$

In order to prove that $T$ has a fixed point $y \in \mathcal{K}_{R}$, we need to verify that $T$ maps $\mathcal{K}_{R}$ continuously into a relatively compact subset of $\mathcal{K}_{R}$.

If $y \in \mathcal{K}_{R},(7)$ implies that $T(y)(1)=0$ and

$$
0 \leq(T y)^{\prime}(r)=\left[\int_{1}^{r} n t^{n-1} F\left(t, y(t), y^{\prime}(t)\right) d t\right]^{\frac{1}{n}}
$$

We shall need to verify that we can find a constant $R>0$ such that

$$
\left[\int_{1}^{+\infty} n s^{n-1} F\left(s, y(s), y^{\prime}(s)\right) d s\right]^{\frac{1}{n}} \leq R, \forall y \in \mathcal{K}_{R}
$$

* If $F$ satisfies $\left(H_{1}\right)$, we can write using $\left(H_{1}\right)(i)$,

$$
(T y)^{\prime}(r) \leq\left[\int_{1}^{r} n s^{n-1} F(s, 0,0) d s\right]^{\frac{1}{n}}
$$

by $\left(H_{1}\right)(i i)$, it suffices then to take

$$
R=\left[n \int_{1}^{+\infty} s^{n-1} F(s, 0,0) d s\right]^{\frac{1}{n}}
$$

and we get

$$
(T y)^{\prime}(r) \leq R
$$

* When $F$ satisfies $\left(H_{2}\right)$, then, since

we get by (6),

$$
y(r)=\int_{1}^{r} y^{\prime}(t) d t,
$$


so,

$$
|y(r)| \leq(r-1) R
$$

$$
(T y)^{\prime}(r) \leq\left[\int_{1}^{r} n C_{0} s^{-\alpha-1}(s-1)^{\beta} R^{\beta+\theta} d s\right]^{\frac{1}{n}} \leq\left(\frac{n}{\alpha-\beta} C_{0}\right)^{\frac{1}{n}} R^{\frac{\beta+\theta}{n}}
$$

In order to get (8), it suffices to take $R$ small enough when $(\beta+\theta)>n$, big enough when $(\beta+\theta)<n$. In the case when $\beta+\theta=n$ and $C_{0} \leq \frac{\alpha-\beta}{n}$, any positive constant $R$ lead to

$$
(T y)^{\prime}(r) \leq R
$$

* Finally, if $F$ satisfies $\left(H_{3}\right)$, then, assumption $\left(H_{3}\right)(i)$ shows that

$$
(T y)^{\prime}(r) \leq\left[\int_{1}^{r} n s^{n-1} F(s,(s-1) R, R) d s\right]^{\frac{1}{n}}
$$

it suffices then to take $R=a$ to ensure by $\left(H_{3}\right)(i i)$ the inequality (8).

To establish the continuity of $T$, let $\left(y_{k}\right)$ be a sequence in $\mathcal{K}_{R}$ with $\lim _{k \rightarrow+\infty} y_{k}=$ $y \in C^{1}$ in the $C^{1}$-topology. By the dominated convergence theorem, we have then

$$
\lim _{k \rightarrow+\infty} \int_{1}^{r} n s^{n-1} F\left(s, y_{k}(s), y_{k}^{\prime}(s)\right) d s=\int_{1}^{r} n s^{n-1} F\left(s, y(s), y^{\prime}(s)\right) d s
$$

uniformly on $\left[1,+\infty\right.$ [, from which $T y_{k}$ and $\left(T y_{k}\right)^{\prime}$ converge uniformly to $T y$ and $(T y)^{\prime}$, respectively, on compact intervals in $\left[1,+\infty\left[\right.\right.$. this means that $T y_{k}$ converges to $T y$ in the $C^{1}$-topology.

The relative compactness of $T\left(\mathcal{K}_{R}\right)$ is a consequence of Ascoli's Theorem; we need only verify the local uniform boundedness and local equicontinuity of the sets $T\left(\mathcal{K}_{R}\right)$ and $T\left(\mathcal{K}_{R}\right)^{\prime}=\left\{(T y)^{\prime}, y \in \mathcal{K}_{R}\right\}$.

Let us denote $G(t)=n F\left(t, u(t), u^{\prime}(t)\right)$ and $\widetilde{G}(t)=n \widetilde{F}\left(t, u(t), u^{\prime}(t)\right)$.

For every $y \in \mathcal{K}_{R}, 1 \leq t_{1} \leq t_{2}$, the inequality $a^{\frac{1}{n}}-b^{\frac{1}{n}} \leq(a-b)^{\frac{1}{n}}$, true for $a \geq b \geq 0$, implies

$$
\begin{aligned}
(T y)^{\prime}\left(t_{2}\right)-(T y)^{\prime}\left(t_{1}\right) & =\left(\int_{1}^{t_{2}} t^{n-1} G(t) d t\right)^{\frac{1}{n}}-\left(\int_{1}^{t_{1}} t^{n-1} G(t) d t\right)^{\frac{1}{n}} \\
& \leq\left(\int_{t_{1}}^{t_{2}} t^{n-1} G(t) d t\right)^{\frac{1}{n}}
\end{aligned}
$$

* If $F$ satisfies $\left(H_{1}\right)$, then

$$
G(t) \leq n F(t, 0,0)
$$

and

$$
(T y)^{\prime}\left(t_{2}\right)-(T y)^{\prime}\left(t_{1}\right) \leq\left(\int_{t_{1}}^{t_{2}} n t^{n-1} F(t, 0,0) d t\right)^{\frac{1}{n}} \rightarrow 0, \text { as } t_{1}, t_{2} \rightarrow \infty
$$

* If $F$ satisfies $\left(H_{2}\right)$, then,

$$
\begin{aligned}
(T y)^{\prime}\left(t_{2}\right)-(T y)^{\prime}\left(t_{1}\right) & \leq\left(\int_{t_{1}}^{t_{2}} n C_{0} t^{n-1} t^{-n-\alpha}(t-1)^{\beta} R^{\beta+\theta} d t\right)^{\frac{1}{n}} \\
& \leq C_{1}\left(\int_{t_{1}}^{t_{2}} t^{\beta-\alpha-1} d t\right)^{\frac{1}{n}} \rightarrow 0, \text { as } t_{1}, t_{2} \rightarrow \infty
\end{aligned}
$$

* Finally, when $F$ satisfies $\left(H_{3}\right)$, then by (i), since $R=a$,

and

$$
G(t) \leq n F(t,(t-1) a, a)
$$


$(T y)^{\prime}\left(t_{2}\right)-(T y)^{\prime}\left(t_{1}\right) \leq\left(\int_{t_{1}}^{t_{2}} n t^{n-1} F(t,(t-1) a, a) d t\right)^{\frac{1}{n}} \rightarrow 0$, as $t_{1}, t_{2} \rightarrow \infty$

Then, in all these cases, for any compact interval $I$ in $[1,+\infty[$ and arbitrary $\varepsilon>0$, there is a corresponding $\delta>0$, independent of $t_{1}, t_{2}$ and $y \in \mathcal{K}_{R}$, such that

for all $t_{1}, t_{2} \in I$ with $\left|t_{1}-t_{2}\right|<\delta$.

$$
\left|(T y)^{\prime}\left(t_{2}\right)-(T y)^{\prime}\left(t_{1}\right)\right| \leq \varepsilon
$$

The local equicontinuity of $T\left(\mathcal{K}_{R}\right)$ can be verified in the same way, and the local uniform boundedness is obvious.

Therefore the Schauder-Tychonoff fixed point theorem ([5]; lemma 1 and [6]; Theorem 4.5.1.) implies that $T$ has a fixed point $u \in \mathcal{K}_{R}$, satisfying the integrodifferential equation (3) for any $R$ such that (8) holds. It remains to prove that $u^{\prime} \in C^{1}$.

For $t>1$, we have

$$
u^{\prime}(t)=\left[\int_{1}^{t} s^{n-1}(s-1)^{l} \widetilde{G}(s) d s\right]^{\frac{1}{n}}
$$

Since $\widetilde{G}(1) \neq 0$, then $\left.u^{\prime} \in C^{1}\right] 1,+\infty[$ and

$$
\begin{aligned}
& u^{\prime \prime}(t)=t^{n-1}(t-1)^{l} \widetilde{G}(t)\left[\int_{1}^{t} s^{n-1}(s-1)^{l} \widetilde{G}(s) d s\right]^{\frac{1}{n}-1} \\
& =t^{n-1}(t-1)^{\frac{l+1}{n}-1} \widetilde{G}(t)\left[\int_{0}^{1}[(t-1) s+1]^{n-1} s^{l} \widetilde{G}((t-1) s+1) d s\right]^{\frac{1}{n}-1}
\end{aligned}
$$

which gives

$$
\lim _{t \rightarrow 1^{+}} u^{\prime \prime}(t)= \begin{cases}0, & \text { if } l>n-1 \\ {\left[\frac{1}{l+1}\right]^{\frac{1}{n}-1} \widetilde{G}(1)^{\frac{1}{n}}} & \text { if } l=n-1\end{cases}
$$

Hence, $u \in C^{2}[1,+\infty$ [. It is not to be noted that $u$ is a solution of (1) satisfying $u(1)=0$ and $u^{\prime}(1)=0$.

Furthermore, the relation (3) and the inequality (8) imply that the limit

$$
\lim _{t \rightarrow+\infty} \frac{u(t)}{t}=\lim _{t \rightarrow+\infty} u^{\prime}(t)=\left[\int_{1}^{+\infty} n s^{n-1} F\left(s, u(s), u^{\prime}(s)\right) d s\right]^{\frac{1}{n}}
$$

is positive and finite, proving the asymptotic property in theorem A.

Since any non-negative constant $b$ will serve as initial value $y^{\prime}(1)=b$, there exists an infinitude of radial convex solutions to our problem.

3. Proof of theorem B. In this section, we study the regularity of the solution $u$ given by theorem $\mathrm{A}$. To prove the $C^{k+2}$ regularity of $u$, let us proceed by induction on $k \in \mathbb{N}$. For $k=0$, we have established in section 2 , that $u \in C^{2}$. Suppose that

$$
\widetilde{F} \in C^{k-1} \Rightarrow u \in C^{k+1}
$$

for some fixed $k \geq 1$. Assume now that $\widetilde{F} \in C^{k}$. It follows in particular that $u \in C^{k+1}$. Hence, from the integral formula (7) and the hypothesis $\left(H_{4}\right)$, we get $u \in C^{k+2}(] 1,+\infty[)$. It remains to check the regularity of $u$ at the boundary $t=1$.

The following preliminary result will be needed

Lemma ([4] corollary 4.2). The $\mathrm{k}^{\text {th }}$ derivative of $g^{\frac{1}{n}}$, can be written as a sum of terms of the form

$$
g^{\frac{1}{n}-\lambda} P_{\lambda}\left(g^{\prime}, g^{\prime \prime}, \ldots, g^{(k+1-\lambda)}\right)
$$


where $P_{\lambda}$ is a monomial of degree $\lambda \leq k$ and of weighted degree $k$.

Now, using the notation

$$
H_{y}(t)=\int_{0}^{1}[(t-1) s+1]^{n-1} s^{l} \widetilde{G_{y}}((t-1) s+1) d s,
$$

we can write

$$
u^{\prime}(t)=(t-1)^{\frac{l+1}{n}} H_{u}^{\frac{1}{n}}(t)
$$

where, by the induction hypothesis, $H_{u} \in C^{k}$. Then,

$$
u^{(k+1)}(t)=\sum_{i=0}^{k}\left(\begin{array}{c}
k \\
i
\end{array}\right)\left[(t-1)^{\frac{1+l}{n}}\right]^{(i)}\left(H_{u}^{\frac{1}{n}}\right)^{(k-i)}(t)
$$

furthermore, applying the above lemma, we get the following

Since, $\forall j \leq k$,

$$
\left(H_{u}^{\frac{1}{n}}\right)^{(l)}=\sum_{i=2}^{l} c_{i} H_{u}^{\frac{1}{n}-i} P_{i}\left(H_{u}^{\prime}, \ldots, H_{u}^{(l+1-i)}\right)+\frac{1}{n} H_{u}^{\frac{1}{n}-1} H_{u}^{(l)}, \quad \forall l \leq k
$$

$$
\left(H_{u}\right)^{(j)}(t)=\sum_{i=0}^{j} c_{i, j} \int_{0}^{1}\left([(t-1) s+1]^{n-1}\right)^{(j-i)} s^{l+i}{\widetilde{G_{u}}}^{(i)}((t-1) s+1) d s
$$

it suffices then to prove that

$$
f(t)=(t-1)^{\frac{1+l}{n}} h_{k} \in C^{1}([1,+\infty[)
$$

where $h_{k}(t)=\int_{0}^{1}[(t-1) s+1]^{n-1} s^{l+k} \widetilde{G}_{u}^{(k)}((t-1) s+1) d s$.

Differentiating $f$, yields

which implies

$$
\forall t>1, f^{\prime}(t)=(t-1)^{\frac{1+l}{n}-1}\left[t^{n-1}{\widetilde{G_{u}}}^{(k)}(t)+c h_{k}(t)\right]
$$

$$
\lim _{r \rightarrow 1^{+}} f^{\prime}(t)= \begin{cases}0 & \text { if } l>n-1 \\ {\widetilde{G_{u}}}^{(k)}(1)\left[1+c \int_{0}^{1} s^{l+k} d s\right], & \text { if } l=n-1\end{cases}
$$

Consequently, $f \in C^{1}([1,+\infty[)$. Which completes the proof of theorem B.

Acknowledgement. The author would like to thank the referee for several helpful suggestions.

\section{REFERENCES}

[1] Рн. Delanö̈, Radially symmetric boundary value problems for real and complex elliptic MongeAmpère equations, Journal of Differential equations, 58 (1985), pp. 318-344.

[2] F. Finster And O.C. SChnürer, Hypersurfaces of prescribed Gauss curvature in exterior domains, Calc. Var., 15 (2002), pp. 67-80.

[3] T. Kusano And Ch.A. Swanson, Existence theorems for elliptic Monge-Ampère equations in the plane, Differential and Integral Equations, 3:3 (1990), pp. 487-493.

[4] D. Monn, Regularity of the complex Monge-Ampère equation for radially symmetric functions of the unit ball, Math. Ann., 275 (1986), pp. 501-511. 
[5] A.J.B. Ротter, An elementary version of the Leray-Schauder theorem, J. Lond. Math. Soc., 2:5 (1972), pp. 414-416.

[6] D.R. Smart, Fixed point Theorems, Cambridge Tracts in Mathematics, No. 66, Cambridge Univ. Press, London/New York, 1974. 
S. KALLEL-JALLOULI 